\title{
The Pluses and Minuses of Obtaining Measurements from Digital Scans
}

\author{
Ravindra S. Goonetilleke ${ }^{1}$, Channa P. Witana ${ }^{1}$, Jianhui Zhao ${ }^{2}$, and Shuping Xiong ${ }^{3}$ \\ ${ }^{1}$ Department of Industrial Engineering and Logistics Management, \\ Hong Kong University of Science \& Technology, Clear Water Bay, Hong Kong \\ ${ }^{2}$ Computer School, Wuhan University, Wuhan, Hubei, PR China \\ ${ }^{3}$ Department of Industrial Engineering and Management, \\ Shanghai Jiao Tong University, Shanghai, PR China \\ ravindra@ust.hk
}

\begin{abstract}
Digital scanners are commonplace and are used in many different applications to obtain three-dimensional shapes and linear and circumferential measurements. Even though scanners can be highly accurate, measurements obtained from scanners can vary depending on how an object is scanned, aligned and processed. In this study, we examined the effect of three different alignment methods of foot scans and their effects on ten different measurements. Variations among methods in capturing foot length are relatively small relative to arch length. The foot girths can be quite sensitive to the registration process depending on the complexity of the algorithms used. As expected, linear and girth measurements based on anatomical landmarks will always be independent of any registration process and are thus good ways to obtain repeatable measurements.
\end{abstract}

Keywords: Scanning, foot, measurement, registration, alignment, brannock, width, girth.

\section{Introduction}

The length and sometimes the width of feet are used to select footwear. There are many studies on foot anthropometry that describe different techniques to measure critical dimensions on feet. These include Freedman et al. [3], Hawes and Sovak [4] and Kouchi [5]. With the availability of cheap computer power and powerful scanning technologies, many researchers are using automatic methods to obtain foot measurements. The required measures can be obtained from the relevant points in the scanned data using feature recognition techniques or by placing markers and identifying anatomical points $[6,8]$. In recent years, there has been an exponential growth in laser scanner technologies for varying applications that claim accuracy within $1 \mathrm{~mm}$. The accuracy of the scanner itself can vary depending on the object that is scanned and the method used for scanning.

Even though scanner accuracy can be within $1 \mathrm{~mm}$, one fundamental issue that has not been studied in relation to foot anthropometrics is the measurement axes. Witana 
et al. [8] found differences between manual measurements, simulated measurements obtained user-generated computer algorithms and commercially available measurement software. They concluded that some of the difference may be attributed to differing registration processes in the differing methods. Researchers and footwear fitters have long used differing orientations and axes to measure feet. For example, the Brannock device (www.Brannock.com), which has been a tool used for measuring feet in shoe stores in the US for a number of years, has a foot length axis that is 38.1 $\mathrm{mm}$ (1.5 inches) from the medial side of the first metatarsal head. Most researchers use another foot axis, the line joining the pternion and the tip of the second toe [5]. This axis can be very problematic in the presence of bunions as the second toe will be deflected from its neutral position, resulting in possibly longer lengths and wider widths. Freedman et al. [3] and Yavatkar [10] proposed a different axis to measure foot flare. This axis is determined by joining the mid-point of the lines located 10 $\mathrm{mm}$ and $50 \mathrm{~mm}$ from the pternion. The objective of this study is to investigate the effects of using different axes for registration especially when determining foot measurements from digital scans to identify the pros and cons of using scans for foot measurements.

\section{Methodology}

\subsection{Participants}

To account for variations in anatomical structures, twenty-five males and twenty-five females were recruited for this experiment. None of them had any visible foot abnormalities or foot illnesses. Their ages ranged between 19 to 24 years with an average age of 21.5 years. The range of foot length was from $210 \mathrm{~mm}$ to $283 \mathrm{~mm}$ with an average of $245 \mathrm{~mm}$.

\subsection{Experimental Procedure}

Each participant's left foot was laser scanned using a Yeti foot scanner (www.vorum.com). Prior to the scan, seven anatomical landmarks were identified and marked on the left foot. Five landmarks were on the top of the metatarsal-phalangeal joints (MPJ), one each at the side of first and fifth MPJ. Then, the participant's left foot was scanned with half body-weight on each foot. The point cloud of data obtained from the scanner including the seven landmarks were stored and processed as described by Witana et al. [8]. The software program developed by Witana et al. [8] has the capability to generate measures for differing foot registrations. It would be tedious to determine the measures for differing alignments with manual measurements. In this paper, we evaluate three different registrations for ten linear and circumferential foot measurements.

The dependent variables were ten left-foot measurements taken on 50 participants' digitally scanned foot shapes. The independent variable was the registration method at three levels: heel centerline, a simulated Brannock device alignment and a pternion 
to second metatarsal head alignment. The latter method, as opposed to the pternionsecond toe tip, has the ability to minimize effects of bunions.

\subsection{Foot Alignment Methods}

The Heel Centerline (HCL) Method. In this method (pictured in Fig. 1), a part of the rear-foot is used to compute the heel centerline, which is thereafter used as the axis for foot measurement. The algorithm for the HCL method is as follows:

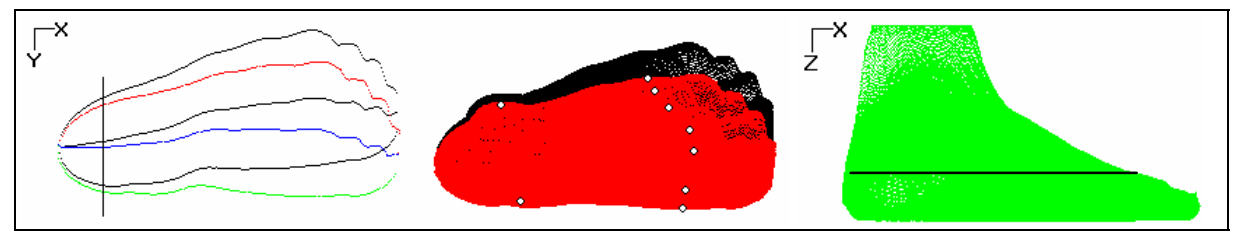

Fig. 1. Heel centerline foot alignment method

1. Find the point, $\mathrm{P}$, with maximum $\mathrm{Z}$ value (foot bottom) from the scanned point cloud (Figure 1).

2. Obtain a data set consisting of points no more than $3 \mathrm{~mm}$ distance from the point, $\mathrm{P}$, along the Z-axis.

3. Find the bottom most point as the mean of the five points with maximum Z-values.

4. Consider all the scan points of the foot that are less than $25 \mathrm{~mm}$ in height (along the negative $\mathrm{Z}$-axis) from this bottom most point.

5. Project the points, determined in Step 4, to the XY plane.

6. Find the two edge points (i.e., points with minimum and maximum $Y$ values) in every scanned section.

7. The center point is then the mean of the two edge points in every section.

8. Fit a least squares line $\left(1^{\text {st }}\right.$ degree polynomial $)$ for all center points that are within $13 \%$ of the foot length from the heel (the first center point).

9. Rotate the foot scanned points around the first center point and parallel to the XY plane to make the fitted line parallel to the X-axis.

10. Repeat Steps 4 to 9 until the rotational angle is less than $0.001 \mathrm{deg}$.

11. Save the rotated results as an aligned point cloud and landmarks.

The Brannock Alignment (BRN) Method. This alignment method (pictured in Fig. 2) simulates the foot positioning in the Brannock device (www.brannock.com). The algorithm used in the BRN alignment method is as follows:

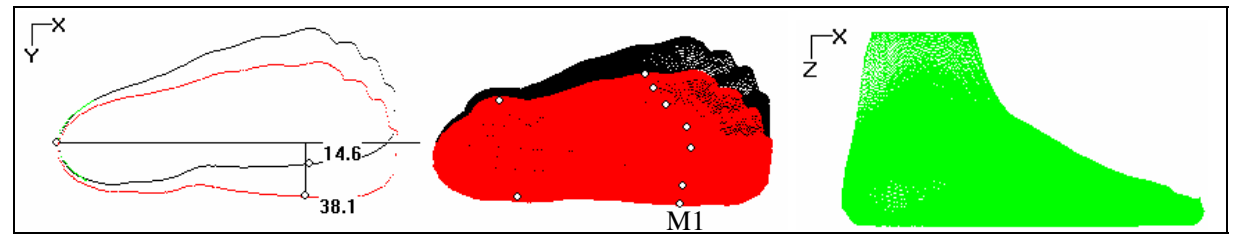

Fig. 2. Foot registration to simulate the Brannock device alignment 
1. Find the point, $P$, with maximum $Z$ value (foot bottom) from the scanned point cloud.

2. Obtain a data set consisting of points no more than $3 \mathrm{~mm}$ distance from the point, $\mathrm{P}$, along the Z-axis.

3. Find the bottom most point as the mean of the five points with maximum $\mathrm{Z}$-values.

4. Consider all the scanned points of the foot that are less than $25 \mathrm{~mm}$ in height (Zaxis) from this bottom most point.

5. Project the points, determined in Step 4, to the XY plane.

6. Find the two edge points (i.e., points with minimum and maximum $Y$ values) in every scanned section.

7. The center point is then the mean of the two edge points in every section.

8. Fit a second-degree polynomial line $\left(x=a y^{2}+b y+c\right)$ for all the edge points that are $25 \mathrm{~mm}$ from the heel along the $\mathrm{X}$ direction.

9. Calculate the turning point coordinates, $\left(\left(4 a c-b^{2}\right) /(4 a),-b /(2 a)\right)$.

10. Calculate the distance from the first landmark (M1) to the line, which is parallel to the $\mathrm{X}$-axis and passing through the turning point.

11. Rotate the foot around the above turning point and parallel to the XY plane to make the distance calculated in step 10 be $38.1 \mathrm{~mm}$, as shown in Figure 2.

12. Save the rotated results as the aligned point cloud and landmarks.

The Pternion-Second Metatarsal Alignment (2MT) Method. This method, pictured in Fig. 3, uses the pternion and a landmark on the second metatarsal. The algorithm used in this method is as follows.

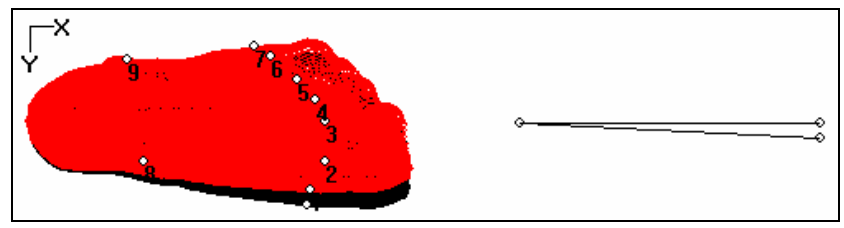

Fig. 3. Foot alignment method based on the pternion and a landmark on the second metatarsal (landmark 3)

1. Find the point, A, with the minimum $X$ value from the scanned point cloud.

2. Obtain a data set (D3) consisting of all points no more than $3 \mathrm{~mm}$ away from the point, A, along the $\mathrm{X}$-axis.

3. Find the five points with the lowest X-values from the data set, D3. Let the mean of these five points represent the pternion, i.e., the backmost point on foot.

4. Calculate the angle between the X-axis and the line (L1) joining the pternion and the second metatarsal head landmark.

5. Rotate the foot point cloud around pternion such that line L1is parallel to the Xaxis, as shown in Figure 3. 


\subsection{Foot Measurements}

Ten foot measurements were obtained using a C++ program. The description of each measurement is given in Table 1 and each measure is shown in Figure 4.

Table 1. Description of the foot metrics. The numbers correspond to those shown in Figure 4.

\begin{tabular}{|c|c|}
\hline \multirow{3}{*}{ Lengths } & $\begin{array}{l}\text { [1] Foot length: The distance along the alignment axis (X- } \\
\text { direction) from the pternion to the tip of the longest toe. }\end{array}$ \\
\hline & $\begin{array}{l}\text { [2] Arch length: The distance along the alignment axis from the } \\
\text { pternion to the most medially prominent point on the first } \\
\text { metatarsal head. }\end{array}$ \\
\hline & $\begin{array}{l}\text { [3] Heel to } 5^{\text {th }} \text { toe: The distance along the alignment axis from } \\
\text { the pternion to the anterior fifth toe tip. }\end{array}$ \\
\hline \multirow{3}{*}{ Widths } & $\begin{array}{l}\text { [4] Foot width: Maximum horizontal breadth (Y-direction), } \\
\text { across the foot perpendicular to the aligned axis in the region } \\
\text { in front of the most laterally prominent point on the fifth } \\
\text { metatarsal head. }\end{array}$ \\
\hline & $\begin{array}{l}\text { [5] Mid-foot width: Maximum horizontal breadth, across the } \\
\text { foot perpendicular to the alignment axis at 50\% of foot } \\
\text { length from the pternion. }\end{array}$ \\
\hline & $\begin{array}{l}\text { [6] Heel width: Breadth of the heel at a location } 40 \mathrm{~mm} \text { anterior } \\
\text { to the pternion (modified from last measurements given by } \\
\text { [7]). }\end{array}$ \\
\hline \multirow{4}{*}{ Girths } & $\begin{array}{l}\text { [7] Ball girth: Circumference of the foot, measured with a tape } \\
\text { touching the medial margin of the head of the first metatarsal } \\
\text { bone, the top of the first metatarsal bone and the lateral mar- } \\
\text { gin of the head of the fifth metatarsal bone. }\end{array}$ \\
\hline & $\begin{array}{l}\text { [8] Instep girth: The smallest girth over the middle cuneiform } \\
\text { prominence [2]. }\end{array}$ \\
\hline & $\begin{array}{l}\text { [9] Short heel girth: Minimum girth around the back heel point } \\
\text { and the dorsal foot surface [1] }\end{array}$ \\
\hline & $\begin{array}{l}\text { [10] Long heel girth: The girth from the instep point around the } \\
\text { back heel point }[1,2] \text {. }\end{array}$ \\
\hline
\end{tabular}

\section{Results}

The means and standard deviations for the ten measurements obtained using each of the alignments on the 50 participants are shown in Table 2. The foot length, arch length and foot width measures for each of the participants using each method are shown in Figures 5, 7 and 9. The differences between methods are presented in Figures 6,8 and 10 . 

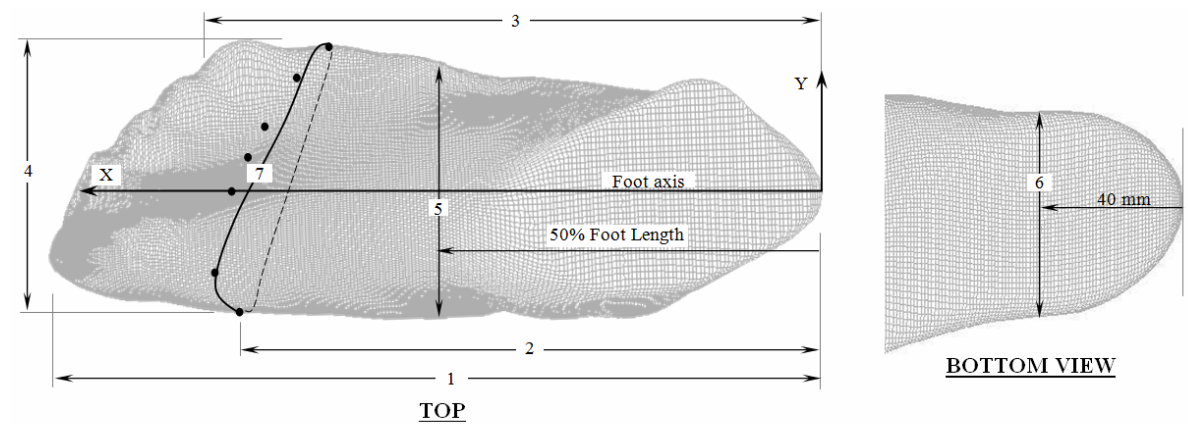

BOTTOM VIEW

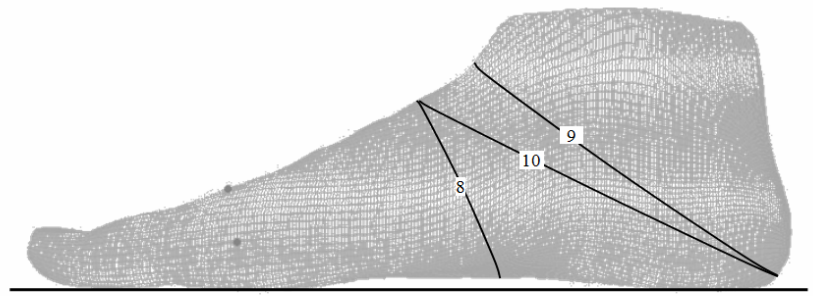

SIDE VIEW

Fig. 4. Foot measurements

Table 2. The means for each of the three alignment methods. The standard deviations are given in parentheses.

\begin{tabular}{lccc}
\hline Foot measurement & HCL & BRN & 2MT \\
\hline Foot Length & $245.0(14.74)$ & $245.3(14.85)$ & $245.0(14.72)$ \\
Arch Length & $178.8(10.50)$ & $180.1(10.80)$ & $179.0(10.44)$ \\
Heel to Fifth Toe & $203.9(14.20)$ & $203.1(13.92)$ & $203.9(14.39)$ \\
Foot Width & $92.6(7.07)$ & $92.6(7.12)$ & $92.5(7.24)$ \\
Heel Width & $61.4(4.13)$ & $61.4(4.13)$ & $61.4(4.10)$ \\
Mid Foot Width & $85.7(7.74)$ & $85.9(7.94)$ & $85.6(7.71)$ \\
Ball Girth & $223.3(16.03)$ & $223.3(16.03)$ & $223.3(16.03)$ \\
Instep Girth & $238.0(16.75)$ & $237.3(16.50)$ & $238.0(16.41)$ \\
Long Heel Girth & $324.5(22.25)$ & $327.1(21.80)$ & $325.9(22.09)$ \\
Short Heel Girth & $304.7(18.62)$ & $304.8(18.51)$ & $304.8(18.48)$ \\
\hline
\end{tabular}




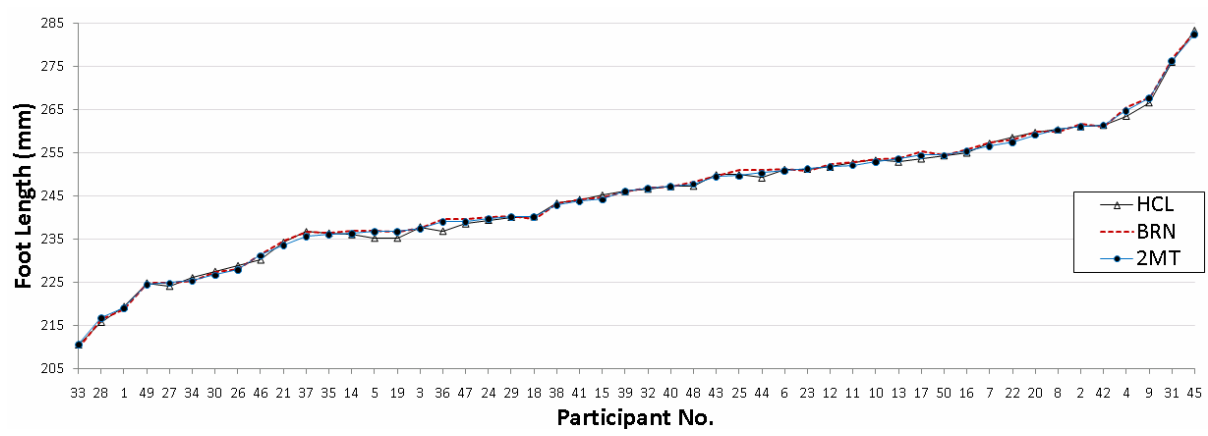

Fig. 5. Foot length measurements using three alignment methods $(n=50)$

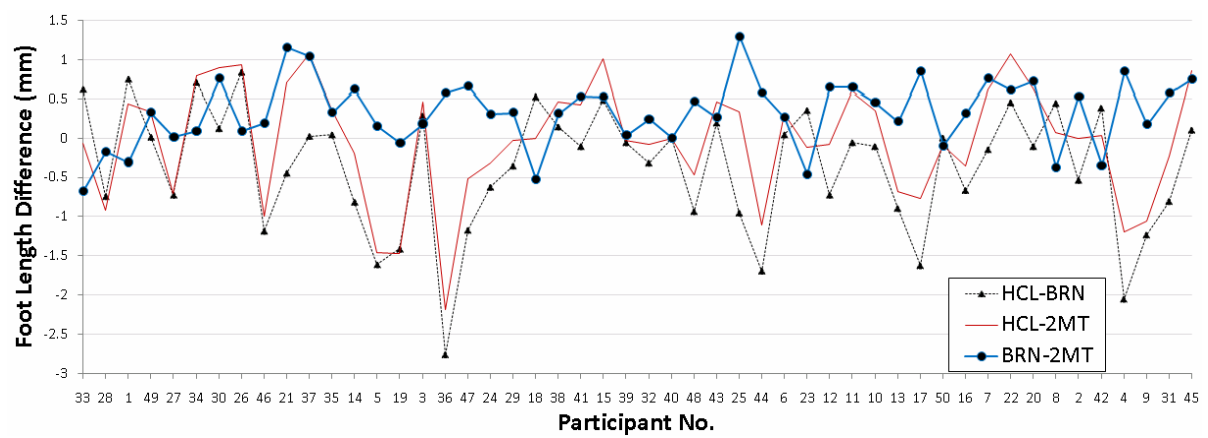

Fig. 6. Differences in foot length measurements among three alignment methods $(n=50)$

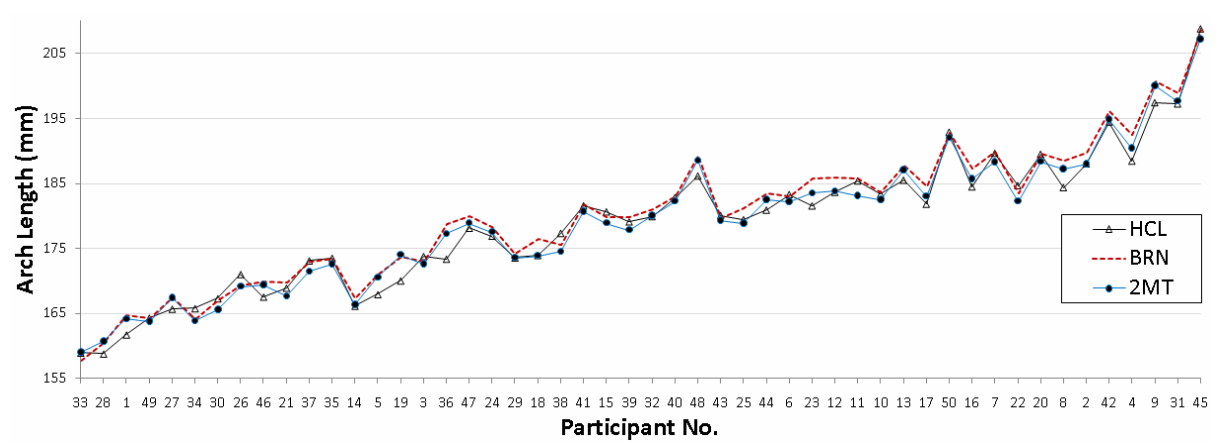

Fig. 7. Arch length measurements using three alignment methods $(n=50)$ 


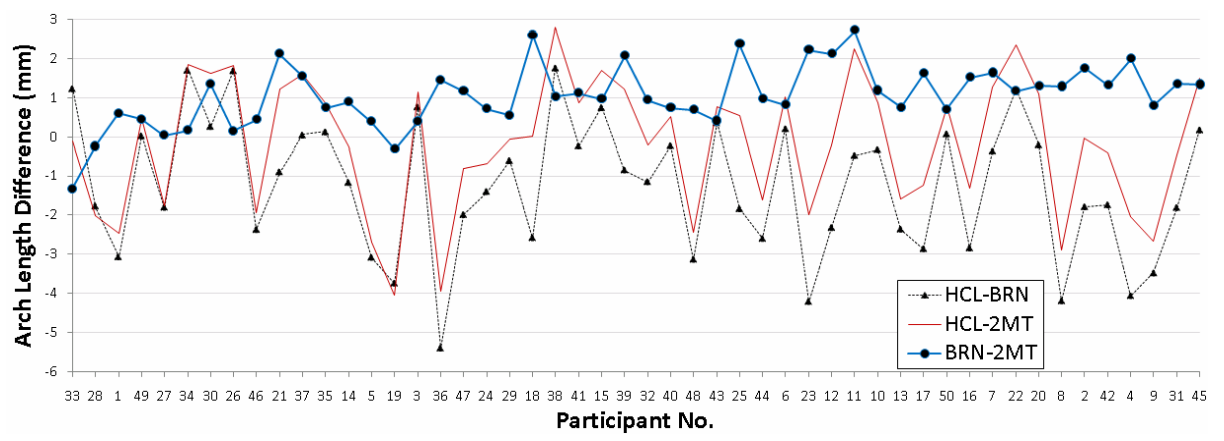

Fig. 8. Differences in arch length measurements among three alignment methods $(n=50)$

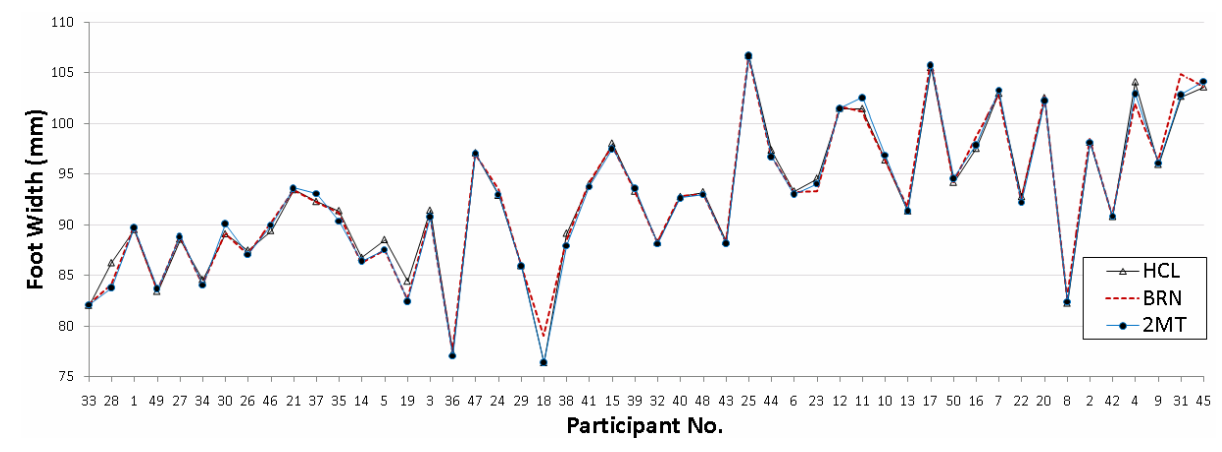

Fig. 9. Foot width measurements using three alignment methods $(n=50)$

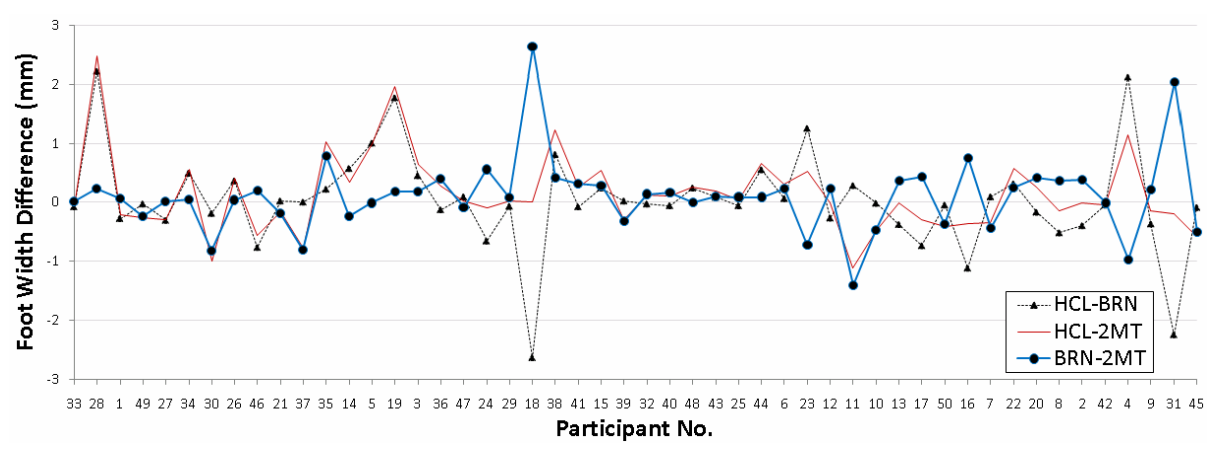

Fig. 10. Differences in foot width measurements among three alignment methods $(n=50)$

\section{Discussion}

The results clearly show that the alignment method has a relatively small effect on measures such as foot length. The maximum difference among the three methods was 
less than $3 \mathrm{~mm}$ for all 50 participants. However, there are differences of about $5 \mathrm{~mm}$ in the different methods in measuring arch length. The differences between the Brannock alignment and the $2 \mathrm{MT}$ alignment methods are relatively small. The differences in foot length and arch length measurements using these two methods are $1.5 \mathrm{~mm}$ and $3 \mathrm{~mm}$, respectively. The heel centerline method of alignment tends to have larger differences with the other two methods of alignment. This is primarily because the alignment is based on the center of the heel. Calluses and other deformities can significantly affect the orientation of the foot axis. The orientation of the foot using this method can be quite different with the other two methods. The arch length is generally around $73 \%$ of foot length [9] and one may expect the differences among methods to be around $73 \%$ of the differences in foot length. However, the larger difference between methods in measuring arch length is due to the projection of the first metatarsal point (arch point) on the measurement axis. Consider any two alignment methods. If the angle between the two alignments is $\theta$, then the difference in foot length (FL) between the two methods is FL $(1-\cos \theta)$. If the straight line distance between the pternion and the first metatarsal head is $y$, and the angle subtended by this line to the alignment axis is $\psi$, then the difference in the arch length between the two methods is $y\{\cos \psi-\cos (\psi+\theta)\}$. Foot length is a special case where $\psi=0$. It can be shown that $\{\cos \psi-\cos (\psi+\theta)\}$ is larger than $\{1-\cos \theta\}$ for $0<\psi<90$, and hence the difference in arch length between two methods will tend to be larger than the difference in foot length.

Variations in width tend to be proportional to the magnitude of the distance; the larger the magnitude, the larger the difference. For example, the differences between methods in measuring heel width tend to be smaller than those measuring foot width. The ball girth had no effect on the alignment as it is based on anatomical landmarks. In other words, the use of anatomical landmarks will cause all measurements to be independent of the alignment of the foot. The data show that there were variations between the alignment methods in the measures of the instep girth and the long heel girth. These differences may be attributed to the locations used for the calculations in the software. Certain anatomical features were used to calculate these girths and their orientations can shift in the calculations. Measurements of the short heel girth, on the other hand, varied less among the different methods as the measuring plane was defined based on the pternion and the minimum value for the girth measurement. The measurement algorithm automatically took care of any variations due to the different alignments. Overall, caution is required when setting the foot axis and obtaining measures based on the axis used.

\section{Conclusion}

Scanning technologies are able to yield good point cloud data. Measurements from the point clouds produced by the scanning can be sensitive to the alignment method. When the measurement points are close to the measurement axis, the variations among alignment methods may be small compared to measurements that are based on points that are further away from the measurement axis. If axis-independent measures are desired, then the ideal scanning situation would be to calculate distances or girths based on anatomical landmarks. 


\section{Acknowledgements}

The authors would like to thank the Research Grants Council of Hong Kong for funding this study under grant HKUST 613607.

\section{References}

1. Chen, C.C.: An investigation in to shoe last design in relation to the foot measurement and shoe fitting for orthopedic footwear, Ph.D. Thesis, University of London (1993)

2. Clarks, Ltd. Training Dept., Manual of shoemaking, 2nd edn., Training Department Clarks (1976)

3. Freedman, A., Huntington, E.C., Davis, G.C., Magee, R.B., Milstead, V.M., Kirkpatrick, C.M.: Foot dimensions of soldiers (Third Partial Report Project No. T 13). Armored Medical Research Laboratory, Fort Knox (1946)

4. Hawes, M.R., Sovak, D.: Quantitative morphology of the human foot in a north American population. Ergonomics 37(7), 1213-1226 (1994)

5. Kouchi, M.: Inter-generation differences in foot morphology: Aging or secular change? Journal of Human Ergology 32, 23-48 (2003)

6. Luximon, A., Goonetilleke, R.S., Tsui, K.L.: Foot landmarking for footwear customization. Ergonomics 46(4), 364-383 (2003)

7. Pivečka, J., Laure, S.: Practical handbook for shoe designers: The shoe last. International school of modern shoemaking (1995)

8. Witana, C.P., Xiong, S., Zhao, J., Goonetilleke, R.S.: Foot measurements from threedimensional scans: a comparison and evaluation of different methods. International Journal of Industrial Ergonomics 36(9), 789-807 (2006)

9. Xiong, S., Goonetilleke, R.S., Zhao, J., Li, W., Witana, C.P.: Foot deformations under different load bearing conditions and their relationships to stature and body weight. Anthropological Science (2009) (in press)

10. Yavatkar, A.S.: Computer aided system approach to determine the shoe-last size and shape based on statistical approximated model of a human foot. Unpublished master's thesis, Tufts University, Medford MA (1993) 